\title{
Prognostic Significance of Serum Uric Acid Levels in Congestive Cardiac Failure And Its Correlation With Ejection Fraction
}

\author{
Dr.S Suresh ${ }^{1}$, Dr.M Palaniappan ${ }^{2}$, Dr.P Praveen Kumar ${ }^{3}$,Dr B Vetriveeran ${ }^{4}$, \\ Dr T Ravikumar ${ }^{5}$,Dr P Vishnuram ${ }^{6}$. Dr. Isaac Christian Moses $^{7}$ \\ ${ }^{1,2}$ Assistant Professor, Dept. Of Medicine, Govt. Medical College \& ESIC Hospital, Coimbatore. \\ ${ }^{3}$ Junior Resident In General Medicine, Coimbatore Medical College Hospital, Coimbatore. \\ ${ }^{4}$ Associate Professor, Dept. Of Medicine, Govt. Medical College, Dharmapuri. \\ ${ }^{5}$ Professor, Dept. Of Medicine, Govt. Medical College \& ESIC Hospital, Coimbatore. \\ ${ }^{6}$ Senior Assistant Professor, Dept. Of Medicine, Coimbatore Medical College, Coimbatore. \\ ${ }^{7}$ Professor, Dept. of Medicine, Coimbatore Medical College, Coimbatore.
}

\begin{abstract}
Introduction: Serum uric acid levels are increased in CHF mostly by increased generation and partly by reduced excretion or both. Elevated uric acid levels indicate cardiac dysfunction and progression of heart failure through oxidative stress and free radical injury by increased xanthine oxidase activity. Epidemiological studies have shown that increased uric acid levels serve as a valid prognostic marker in congestive heart failure and indicate metabolic, functional and hemodynamic derangements. The purpose of this study is to determine the association between uric acid levels and heart failure and to find its significance in predicting mortality and severity of the disease as a prognostic risk marker.

Methodology: The study was conducted in Coimbatore medical college hospital, Coimbatore, which included 100 patients who were admitted with features of heart failure. Patients, who satisfied the criteria for inclusion, were subjected to basic blood investigations and serum uric acid levels were measured in them. Echocardiography was done in all the patients to assess the severity of heart failure. The patients were followed up for a period of one month to determine the mortality and adverse outcomes. The patients were followed up for a period of one month to determine the mortality and adverse outcomes.

Results: Hyperuricemia was found to be significantly higher in patients with cardiac failure and the severity of UA rise had a high correlation to the severity of heart failure. Hyperuricemia is more common in acute decompensated heart failure than chronic heart failure patients. Elevated serum uric acid levels correlate inversely with ejection fraction suggesting that progressive hyperuricemia in cardiac failure indicates deteriorating cardiac function. Patients with elevated serum UA levels were associated with poorer NYHA functional class proving that hyperuricemia predicts the severity of cardiac failure. Hyperuricemia patients were associated with adverse clinical and biochemical features compared to normouricemic patients in the form of increased QRS duration and inotropic requirements. Serum uric acid levels were increased in diabetes and hypertension and had an independent association, irrespective of the etiology of heart failure. Patients with higher uric acid levels were associated with adverse outcomes and poor prognosis in the form of increased rehospitalization rates and 30 day mortality rates.

Conclusion: Thus, it is evident from this study that high serum uric acid levels could be a strong and valid biomarker of impaired prognosis and mortality in patients with cardiac failure, predicting the severity and hemodynamic derangements. The measurement of serum uric acid levels is simple and widely available at low cost. Hence routine measurement of serum uric acid levels in assessing the cardiovascular risks may contribute to the improved ability to stratify risk in cardiac failure.
\end{abstract}

\section{Introduction}

Congestive heart failure (CHF) is an important cause for morbidity and mortality irrespective of age and sex across the globe. Heart failure is associated with poor prognosis leading to frequent hospitalizations, poor quality of life and shortened life span. Hence it is important to identify simple and effective investigatory modalities for early diagnosis, risk stratification and prognosis. Uric acid (UA) is a metabolic end product of purine metabolism. Uric acid appears to play a pathological role in the development of hypertension, diabetes, obesity and metabolic syndrome contributing to cardiovascular mortality. Excess uric acid levels results in endothelial dysfunction, increased RAAS (Renin Angiotensin Aldosterone System) activation and vascular smooth muscle proliferation leading to cardiovascular and renal diseases. ${ }^{1}$ Elevated uric acid levels indicate cardiac dysfunction and progression of heart failure through oxidative stress and free radical injury by increased xanthine oxidase activity. ${ }^{2}$ The purpose of this study is to determine the association between uric acid levels and heart failure and to find its significance in predicting mortality and severity of the disease as a prognostic risk 
marker. The aim of the study is to determine the prognostic significance of serum uric acid levels in patients with congestive cardiac failure and its correlation with ejection fraction.

\section{Materials \& Methodology}

It is a prospective study of 100 patients admitted at Coimbatore Medical College Hospital with Heart Failure during the time period from 1st January 2016 to $31^{\text {st }}$ March 2016. Study was conducted after getting clearance from the ethical committee of our hospital.

\section{Inclusion criteria:}

$>$ Patients admitted with symptoms and signs of Heart Failure

$>$ Age group of both sexes $>18$ years

\section{Exclusion criteria:}

$>$ Acute Coronary Syndromes

$>$ Recent myocardial infarction or angina $(<1$ month $)$

$>$ Chronic obstructive pulmonary disease

$>$ Chronic renal failure $($ Sr. creatinine $>2 \mathrm{mg} / \mathrm{dl})$

$>$ Hematological malignancies

$>$ Already diagnosed hyperuricemia patients

$>$ Patients on uricosuric drugs

\section{Results}

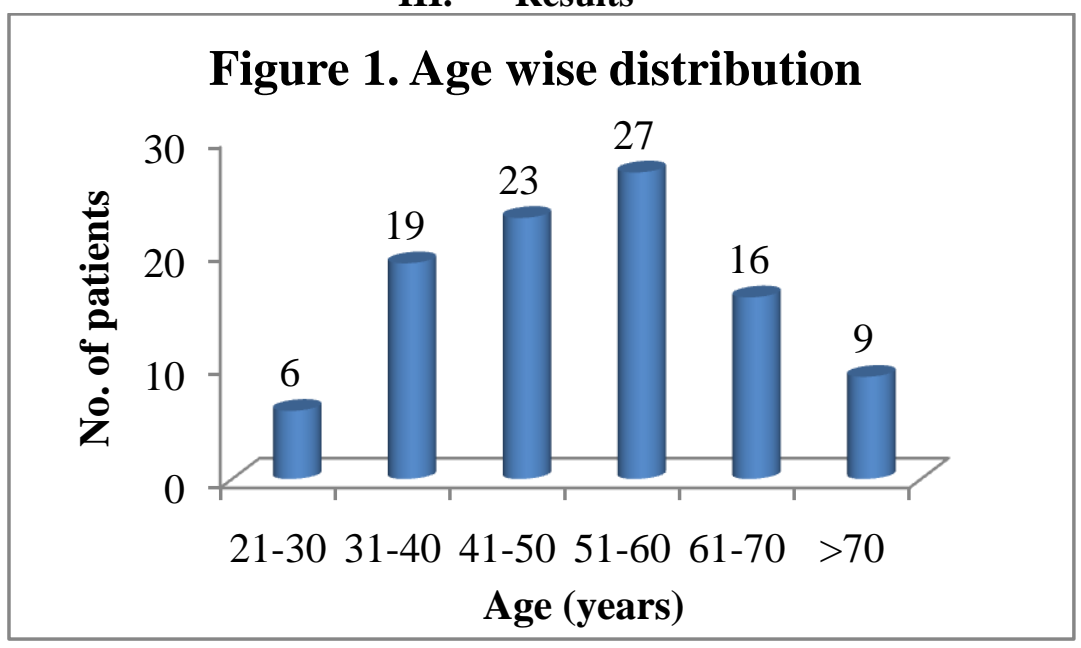

In the study group, majority of the patients were males comprising about $62 \%$ and females about $38 \%$. In this study of 100 patients, majority of the patients were in fourth and sixth decades. The serum uric acid levels were elevated in heart failure with results showing that majority $73 \%$ had hyperuricemia and minority $27 \%$ had normal uric acid levels.

Figure-2 Correlation between Age and Ejection Fraction

\begin{tabular}{|c|c|c|c|c|c|c|c|}
\hline \multirow[b]{2}{*}{ LVEF \% } & \multicolumn{5}{|c|}{ Uric Acid (mg/dl) } & \multirow[b]{2}{*}{$p$ value } & \multirow{2}{*}{$\begin{array}{l}\text { Pearson } \\
\text { Correlation } \\
\text { Coefficient }\end{array}$} \\
\hline & $\leq 6$ & 6.1-7.0 & $7.1-8.0$ & 8.1-9.0 & $>9.0$ & & \\
\hline $\mathbf{2 5 0}$ & 9 & 0 & 0 & 0 & 0 & \multirow{4}{*}{$<0.01$} & \multirow[b]{4}{*}{-0.853} \\
\hline $40-49$ & 10 & 9 & 15 & 7 & 0 & & \\
\hline 30-39 & 0 & 1 & 8 & 21 & 9 & & \\
\hline$<30$ & 0 & 0 & 0 & 3 & 8 & & \\
\hline
\end{tabular}

The serum UA levels were compared with various grades of LVEF. In patients with decreased ejection fraction, all the patients (100\%) had significant rise in serum UA. As the ejection fraction increased, the number of patients with elevated serum uric acid levels proportionately decreased ( $0 \%$ in patients with EF>50). This association holds good with a $\mathrm{P}$ value of $<0.01$ and Pearson correlation coefficient of -0.853 . Thus this study shows that elevated uric acid levels had negative correlation with ejection fraction proving that progressive hyperuricemia indicates cardiac dysfunction in heart failure. 


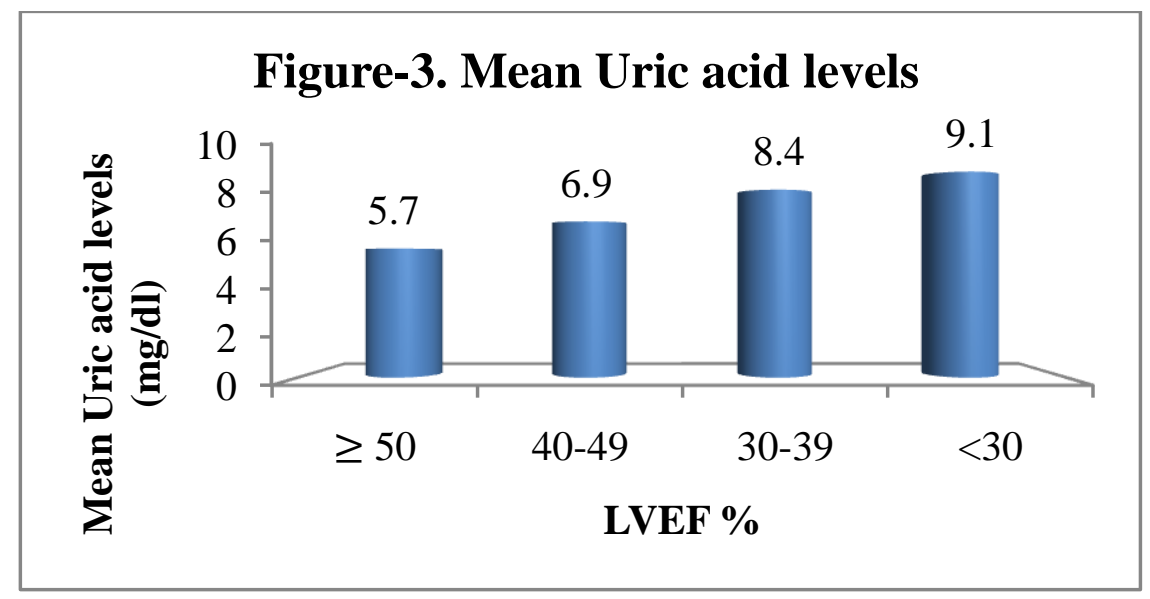

The mean UA levels of patients with different ejection fractions were compared. There was significant rise of serum UA levels in patients with reduced ejection fraction. The mean UA levels of patients with LVEF of $>50 \%, 40-49 \%, 50-59 \%$ and $<30 \%$ are $5.7,6.9,8.4$ and $9.1 \mathrm{mg} / \mathrm{dL}$ respectively. These results also show that serum uric acid levels correlate negatively with ejection fraction in heart failure.

The serum UA levels were compared with various grades of NYHA functional class in HF. In patients with grade III and IV NYHA, majority had significant rise in serum UA. As the NYHA functional class increases, the number of patients with elevated serum uric acid levels proportionately increases. This association holds good with a $\mathrm{P}$ value of $<0.01$. Thus this study shows that elevated uric acid levels had positive correlation with NYHA functional class proving that hyperuricemia indicates progression of the disease in heart failure.

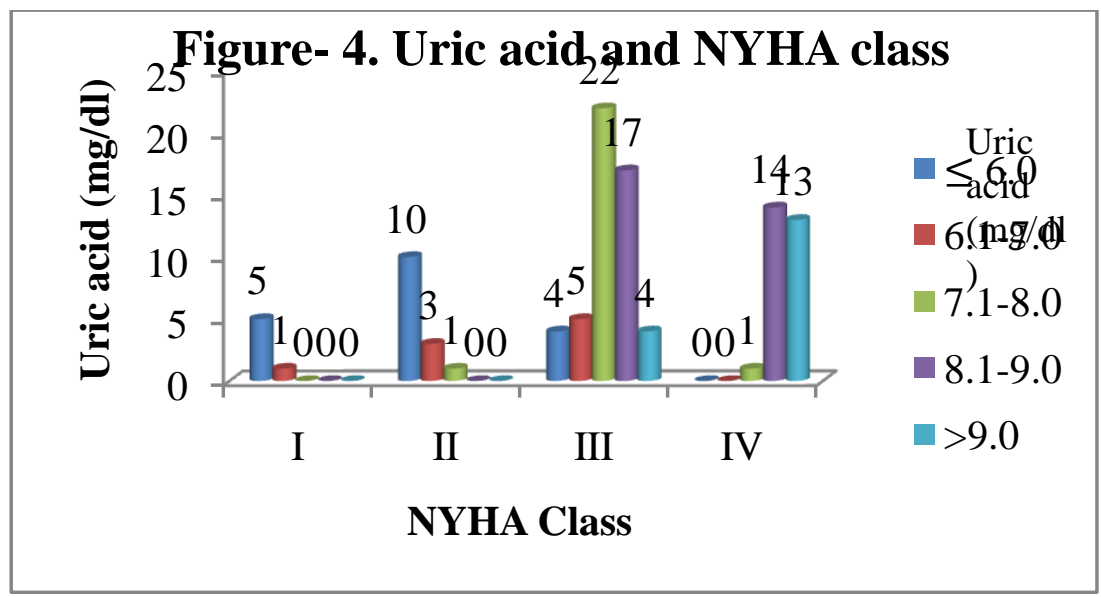

Mean uric acid levels were higher in acute decompensated heart failure than chronic HF showing the significant association of $\mathrm{p}$ value $<0.01$.

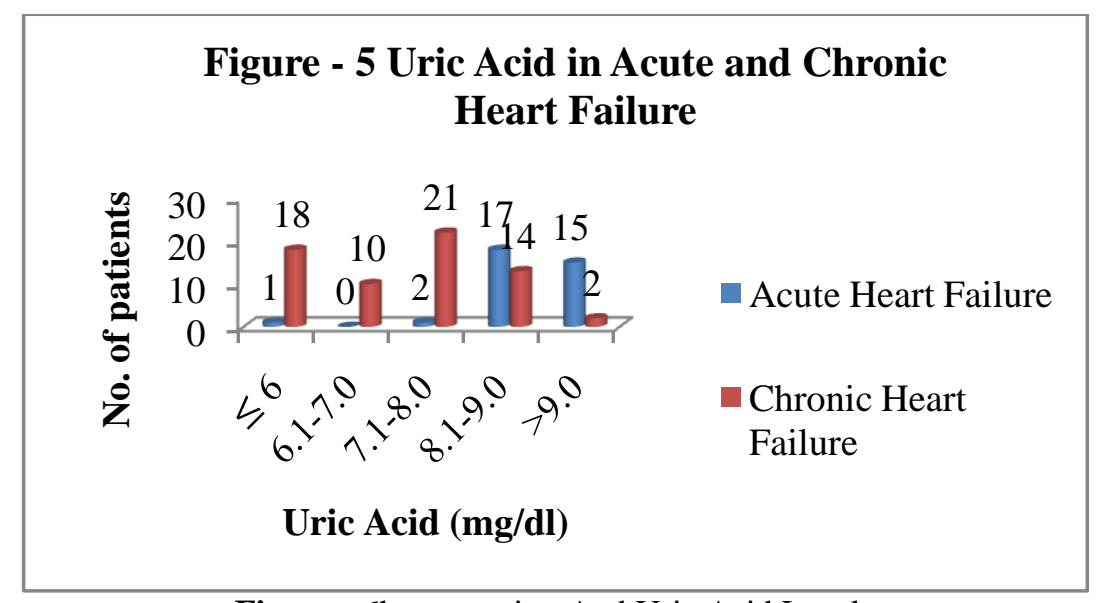

Figure - 6hypertension And Uric Acid Levels 
Prognostic Significance Of Serum Uric Acid Levels In Congestive Cardiac Failure And Its ...

\begin{tabular}{|l|l|l|l|l|l|l|}
\hline \multirow{2}{*}{ Hypertension } & \multicolumn{4}{|l|}{ Uric Acid (mg/dl) } & \multirow{2}{*}{ p value } \\
\cline { 2 - 7 } & $\mathbf{5 6 . 0}$ & $\mathbf{6 . 1 - 7 . 0}$ & $\mathbf{7 . 1 - 8 . 0}$ & $\mathbf{8 . 1 - 9 . 0}$ & $\mathbf{> 9 . 0}$ & \multirow{2}{*}{$<0.01$} \\
\hline Hypertensive & 8 & 2 & 6 & 20 & 11 & \\
\hline Normotensive & 11 & 8 & 17 & 11 & 6 & \\
\hline
\end{tabular}

Serum UA levels were compared in both hypertensive and normotensive individuals. The correlation had a $\mathrm{p}$ value of $<0.01$, which is significant showing that hyperuricemia is associated with hypertension.

Figure- 7 Requirements Of Inotropes To Uric Acid Levels

\begin{tabular}{|c|c|c|c|c|c|c|}
\hline \multirow{2}{*}{ Inotropes } & \multicolumn{5}{|c|}{ Uric Acid (mg/dl) } & \multirow{2}{*}{ p value } \\
\cline { 2 - 6 } & $\mathbf{5 6 . 0}$ & $\mathbf{6 . 1 - 7 . 0}$ & $\mathbf{7 . 1 - 8 . 0}$ & $\mathbf{8 . 1 - 9 . 0}$ & $>\mathbf{9 . 0}$ & \\
\hline Required & 0 & 0 & 1 & 15 & 13 & \multirow{2}{*}{$<0.001$} \\
\hline Not Required & 19 & 10 & 22 & 16 & 4 & \\
\hline
\end{tabular}

In the study, serum uric acid levels were compared with patients who required inotropic supports and those who do not. All patients $(100 \%)$ who required inotropes had elevated UA levels. The correlation had a $\mathrm{p}$ value $<0.001$ which is significant.

Figure-8 re-Hospitalization To Uric Acid Levels

\begin{tabular}{|c|c|c|c|c|c|c|}
\hline \multirow{2}{*}{ Re-hospitalization } & \multicolumn{5}{|c|}{ Uric Acid (mg/d) } & \multirow{2}{*}{ p value } \\
\cline { 2 - 6 } & $\mathbf{5 6 . 0}$ & $\mathbf{6 . 1 - 7 . 0}$ & $\mathbf{7 . 1 - 8 . 0}$ & $\mathbf{8 . 1 - 9 . 0}$ & $\mathbf{> 9 . 0}$ & \multirow{2}{*}{$<0.003$} \\
\hline Yes & 0 & 0 & 0 & 8 & 5 & \multirow{2}{*}{} \\
\hline No & 19 & 10 & 23 & 23 & 12 & \\
\hline
\end{tabular}

In the study, serum uric acid levels were compared with patients with rehospitalisation rates within one month. All patients (100\%) who were rehospitalised had elevated UA levels. The correlation had a $\mathrm{p}$ value $<0.003$ which is significant.

Figure-9 Correlation Of Mortality To Uric Acid Levels

\begin{tabular}{|c|c|c|c|c|c|c|}
\hline \multirow{2}{*}{ Mortality } & \multicolumn{5}{|c|}{ Uric Acid (mg/dl) } & \multirow{2}{*}{ p value } \\
\cline { 2 - 6 } & $\mathbf{5 6 . 0}$ & $\mathbf{6 . 1 - 7 . 0}$ & $\mathbf{7 . 1 - 8 . 0}$ & $\mathbf{8 . 1 - 9 . 0}$ & $\mathbf{> 9 . 0}$ & \\
\hline Yes & 0 & 0 & 0 & 6 & 7 & \multirow{2}{*}{$<0.001$} \\
\hline No & 19 & 10 & 23 & 25 & 10 & \\
\hline
\end{tabular}

In the study, serum uric acid levels were compared with 30 day mortality rate. All patients (100\%) who died within one month had elevated UA levels. The correlation had a p value $<0.001$ which is significant.

\section{Discussion}

Previous studies have shown that serum uric acid may also function as a prognostic risk marker in HF patients. BNP increases as an adaptive response due to increased ventricular filling pressures, whereas uric acid levels are increased in HF as a maladaptive response of increased xanthine oxidase activity. This study shows that hyperuricemia is found in majority $(73 \%)$ of heart failure patients with increased prevalence in females compared to males which is in concurrence with the research studies by Michelle and Uzma et.al in 2009. Serum uric acid levels are increased markedly in acute decompensation than chronic heart failure. Pascual and figal in 2007 shows that hyperuricemia is more common in acute heart failure syndromes than chronic state and majority of the patients with hyperuricemia in acute decompensation shows severe clinical impairment. Increased uric acid levels in acute HF indicates increased oxidative stress and xanthine oxidase activity causing adverse left ventricular remodeling. Endothelial dysfunction and hypoxia causes exaggerated xanthine oxidase activity producing hyperuricemia in heart failure, which is further augmented by the impaired kidney function and use of diuretics in heart failure.

Left ventricular ejection fraction and NYHA functional class were taken as the markers for the severity of heart failure. The study shows that in patients with decreased ejection fraction, all the patients $(100 \%)$ had significant rise in serum UA. As the ejection fraction increased, the number of patients with elevated serum uric acid levels proportionately decreased ( $0 \%$ in patients with $\mathrm{EF}>50)$. This association holds good with a $\mathrm{p}$ value of $<0.01$. Thus this study shows that elevated uric acid levels had negative correlation with ejection fraction proving that progressive hyperuricemia indicates cardiac dysfunction in heart failure.

The severity of heart failure was compared with the severity of uric acid rise, which showed progressive decrease in ejection fraction with increasing uric acid values. This association was statistically significant. The mean uric acid values also increased with decreasing ejection fraction. The mean ejection fraction in patients with hyperuricemia and normal uric acid levels are $36 \%$ and $49 \%$ respectively. Hence, serum 
uric levels can be used as a qualitative marker for assessing the severity of heart failure. These results were confirmative of the research done by Pinelli and Hiroshi et. al in 2007.

The prognosis of patients was assessed by means of 30 day mortality rate and rehospitalization rates within one month. It was found that patients with higher serum uric acid levels were associated with increased rates of rehospitalization and increased mortality rate. The association was statistically significant with a $\mathrm{p}$ value of $<0.001$. Adriana and Danielle et. al in 2008 also proved the same association.

From the above findings, it is made clear that elevated uric acid levels serves as a clinical marker for the multiple pathological processes in the progression of heart failure and reduction in xanthine oxidase activity improves cardiac contractility, reduces oxidative stress, limits infarct size, inflammation and platelet adhesiveness. All these effects would be protective to patients with heart failure and suggests a hypothesis that xanthine oxidase inhibitors may act as novel drugs in the management of cardiac failure in future.

\section{Conclusion}

The prevalence of hyperuricemia was found to be significantly higher in patients with cardiac failure and the severity of UA rise had a high correlation to the severity of heart failure. Hyperuricemia is more common in acute decompensated heart failure than chronic heart failure patients. Elevated serum uric acid levels correlate inversely with ejection fraction suggesting that progressive hyperuricemia in cardiac failure indicates deteriorating cardiac function. . Patients with higher uric acid levels were associated with adverse outcomes and poor prognosis in the form of increased rehospitalization rates and 30 day mortality rates. 